\title{
Petr Veselý
}

Majorants of matrix norms and spectrum localization

Czechoslovak Mathematical Journal, Vol. 44 (1994), No. 1, 141-161

Persistent URL: http://dml.cz/dmlcz/128445

\section{Terms of use:}

(C) Institute of Mathematics AS CR, 1994

Institute of Mathematics of the Czech Academy of Sciences provides access to digitized documents strictly for personal use. Each copy of any part of this document must contain these Terms of use.

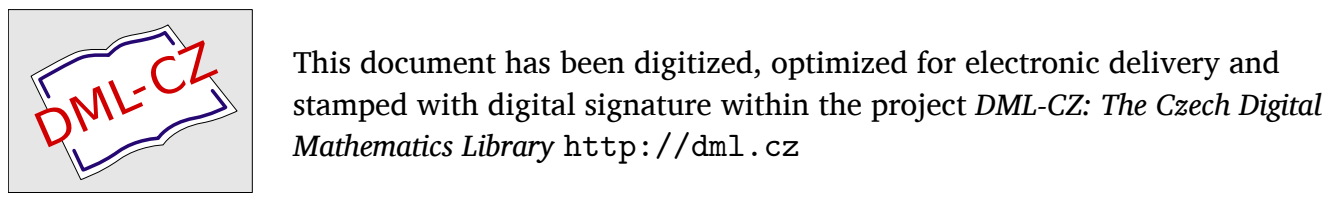




\title{
MAJORANTS OF MATRIX NORMS AND SPECTRUM LOCALIZATION
}

\author{
Pétr Veselý, Praha
}

(Received April 30, 1992)

Let $\mathbf{V}$ be a subspace of $\mathbb{R}^{n}$, let $\mathscr{Q}$ be a set of $n \times n$ real matrices such that $A x \in \mathbf{V}$ whenever $A \in \mathscr{Y}, x \in V$ and let $\tau: \mathscr{W} \rightarrow[0, \infty)$. The paper introduces conditions upon which there exists a vector norm \|\| on $V$ such that

$$
\sup _{\substack{x \in \mathbf{v} \\ x \neq 0}} \frac{\|A x\|}{\|x\|} \leqslant \tau(A) \text { for all } A \in \mathscr{A} .
$$

Moreover, a constructive definition of norms satisfying (1) is presented.

The results are applied to spectrum localization of stochastic matrices in the second part of the paper. The maximum modulus of subdominant eigenvalues is an important characteristic of stochastic matrices. Determination of upper bounds for this value is one of the main objectives of the theory of coefficients of ergodicity, which investigates those of the form

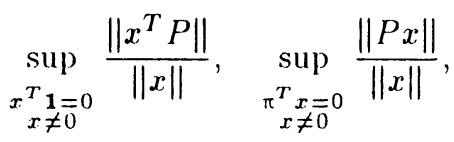

where $P$ is a given stochastic matrix, \|\| is a vector norm, $\pi$ is the stationary distribution of $P$ and $\mathbf{1}=(1,1, \ldots, 1)^{T}$, see $[2,4,6,7,9,10]$. Another upper bound is studied for example in [11].

In this paper, upper bounds of the maximum modulus of subdominant eigenvalues are approached in a more general setting. Namely, a broad subclass $\mathscr{C}$ of the class of all functions $\tau$ satisfying the inequality (1) for $\mathbf{V}=\left\{x \in \mathbb{R}^{n} \mid x^{T} \mathbf{1}=0\right\}$ and for at least one vector norm $\|||$ is taken into consideration. The advantage of this approach is that an upper bound of the maximum modulus of subdominant eigenvalues can be chosen in such a way that both the verification of $\tau \in \mathscr{C}$ and the formula for $\tau(P)$ 
itself are simple in contrast to the calculation of (2) for most of the vector norms \|\| . Moreover, Theorem 2.4 shows that, in the case of an irreducible aperiodic stochastic matrix $P$, any $\tau \in \mathscr{C}$ can be taken to produce an arbitrary tight estimate for the second largest modulus of eigenvalues of the matrix $P$. Examples of suitable functions $\tau \in \mathscr{C}$ are presented as well.

\section{Notation}

$\mathbf{M}_{n}$ - the set of all $n \times n$ real matrices identified with the vector space $\mathbb{R}^{n^{2}}$;

$\langle$,$\rangle - the scalar product on \mathbf{M}_{n}$ defined by

$$
\langle A, B\rangle=\sum_{r=1}^{n} \sum_{s=1}^{n} a_{r s} b_{r s}, \quad A, B \in \mathbf{M}_{n}
$$

$\langle\langle\rangle\rangle$ - the norm on $\mathbf{M}_{n}$ defined by

$$
\langle\langle A\rangle\rangle=\langle A, A\rangle^{1 / 2}, \quad A \in \mathbf{M}_{n}
$$

$\mathscr{S}_{n}$ - the set of all $n \times n$ stochastic matrices;

$\Sigma_{n}$ - the set of all $n \times n$ real matrices such that their columm sums are equal to 1 ;

$\sigma(a)$ - the spectrum of a (square) matrix $A$;

$\varrho(A)$ - the spectral radius of a (square) matrix $A$;

$\operatorname{Lin}(M)$ - the linar span of a set $M \subseteq \mathbb{R}^{n}$;

$I_{n}$ - the $n \times n$ identity matrix;

$\mathbf{0}=(0,0, \ldots, 0)^{T} \in \mathbb{R}^{n}$

$\mathbf{1}=(1,1, \ldots, 1)^{T} \in \mathbb{R}^{n}$.

\section{UPPER BOUNDS FOR NORMS OF MATRIX OPERATORS}

Let $\mathbf{V}$ be a vector subspace of $\mathbb{R}^{n}$ and let $\operatorname{dim} \mathbf{V} \geqslant 1$. Troughout this section, 6 is a set of $n \times n$ real matrices such that

(I) if $A, B \in \mathscr{A}$ and $\alpha \in \mathbb{R}$ then $\alpha A+(1-\alpha) B \in \mathscr{S}$;

(II) if $A, B \in \mathscr{W}$ then $A B \in \mathscr{W}$;

(III) $\operatorname{Lin}(\mathscr{O} \mathbf{V})=\mathrm{V}$;

(IV) there exists at least one matrix $A \in \mathscr{d}$ satisfying $A \mathbf{V}=\{\mathbf{0}\}$.

Let us give some examples. Let $\mathbf{V}=\mathbb{R}^{n}$. Then the set of all $n \times n$ real upper (lower) triangular matrices and the set of all $n \times n$ real matrices with a fixed eigenvector meet the demands of (I), .., (IV). The important set of matrices satisfying the conditions (I), .., (IV) is studied in Section 2 . 
This section is devoted to the study of funcions $\tau: \mathscr{d} \rightarrow[0, \infty)$ satisfying the conditions

$(\alpha) \quad \tau$ is a convex funcion on. $\mathcal{\gamma}^{\prime}$;

(B) $\tau(A B) \leqslant \tau(A) \tau(B)$ for all $A, B \in \alpha$;

$(\gamma)$ there exists at least one matrix $Z \in \mathscr{\alpha}$ such that $\tau(Z)=0$;

$(\delta)$ if $Z \in \mathcal{Q}$ and $\tau(Z)=0$ then $Z \mathbf{V}=\{0\}$;

$(§)$ if $A, Z \in \sigma 8$ and $\tau(Z)=0$ then $\tau(\alpha A+(1-\alpha) Z)=\alpha \tau(A)$ holds for any $\alpha>0$.

Note that a set of functions $\tau: \mathscr{d} \rightarrow[0, \infty)$ satisfying these conditions is noncmpty. Indeed, let || || be a vector norm on $V$ and let

$$
\tau_{\|\|}(A)=\sup _{\substack{x \in \mathbf{V} \\ x \neq 0}} \frac{\|A x\|}{\|x\|}
$$

for every $A \in \mathscr{W}$. It is easily seen that the conditions $(\alpha), \ldots,(\varepsilon)$ hold for the function $\tau_{\|\|}$(the condition $(\gamma)$ holds by virtue of $($ IV $)$ ).

Definition. Let $\mathscr{\gamma}=\left\{v^{(1)}, \ldots, v^{(k)}\right\}$ be a finite subset of $\mathbf{V}$ satisfying $\operatorname{Lin}(\omega \mathcal{V})=\mathrm{V}$, let $\tau$ be a nomnegative function on $\mathscr{d}$ and let $x \in \mathbf{V}$. Define the quantity

$$
[x]_{\mathscr{Y}^{\prime}}^{\tau}=\inf \left\{\sum_{i=1}^{2 k} c_{i} \tau\left(Q_{i}\right) \mid(c, Q) \in \mathscr{K}_{\mathcal{Y}^{\prime}}(x)\right\},
$$

where $\mathscr{K}_{y}(x)$ is the set of all pairs $(c, Q) \in[0, \infty)^{2 k} \times \mathscr{d}^{2 k}$ such that

$$
\sum_{i=1}^{k}\left(c_{i} Q_{i}-c_{k+i} Q_{k+i}\right) v^{(i)}=x .
$$

Romark. The condition (III) implies that there always exists a finite set $\boldsymbol{y}^{\prime} \subset \mathbf{V}$ such that $\operatorname{Lin}(\mathscr{O V})=V$ (for example, any base of $V$ has this property). The reader can verify that, for any such set $\mathscr{Y}^{\prime}$ and for any $x \in \mathrm{V}$, the set $\mathscr{K}_{\mathscr{Y}}(x)$ is non-empty. Thus the quantity $[x]_{\mathcal{Y}}^{\tau}$ is well-defined.

Theorem 1.1. Let $\tau$ be a nonnegative function on a satisfying the conditions $(\alpha)$ and $(\beta)$, let $\boldsymbol{Y}=\left\{v^{(1)}, \ldots, v^{(k)}\right\}$ be a finite subset of $\mathbf{V} \operatorname{such}$ that $\operatorname{Lin}(\alpha \mathscr{Y})=\mathbf{V}$. If $[x]_{\mathscr{Y}}^{\tau}>0$ for each $x \in \mathrm{V}-\{0\}$, then []$_{\mathscr{Y}}^{\tau}$ is a norm on $\mathrm{V}$ and

$$
\operatorname{sip}_{\substack{x \in \mathbb{v} \\ x \neq 0}} \frac{[A x]^{\tau}}{[x]_{\mathscr{Y}}^{\tau}} \leqslant \tau(A)
$$

for all $A \in$ s 
Proof. It is obvious that [ $]_{\mathscr{Y}}^{\tau}$ is a nomnegative function on od and that $[x]^{\tau}=0$ if and only if $x=0$ according to the assumptions.

Let $x \in V$ and let $\lambda>0$. The relation $(c, Q) \in \mathscr{K}_{\mathscr{Y}}(x)$ holds if and only if $(\lambda c, Q) \in \mathscr{K}_{\mathscr{V}}(\lambda x)$. It implies $[\lambda x]_{\mathscr{V}}^{\tau}=\lambda[x]_{\mathscr{V}}^{\tau}$. The relation

$$
\left(\left(c_{1}, \ldots, c_{k}, c_{k+1}, \ldots, c_{2 k}\right),\left(Q_{1}, \ldots, Q_{k}, Q_{k+1}, \ldots, Q_{2 k}\right)\right) \in \mathscr{K}_{Y}(x)
$$

holds if and only if

$$
\left(\left(c_{k+1}, \ldots, c_{2 k}, c_{1}, \ldots, c_{k}\right),\left(Q_{k+1}, \ldots, Q_{2 k}, Q_{1}, \ldots, Q_{k}\right)\right) \in \mathscr{K}_{Y^{\prime}}(-x)
$$

holds. Thus $[x]_{\mathscr{V}}^{\tau}=[-x]_{\mathscr{V}}^{\tau}$. We conclude that $[\lambda x]_{\mathscr{V}}^{\tau}=|\lambda|[x]_{\mathscr{Y}}^{\tau}$ for each $\lambda \in \mathbb{R}$.

Let $x, y \in \mathbf{V}$. If $x=0$ then the triangular inequality $[x]_{Y^{\prime}}^{\tau}+[y]_{Y^{\prime}}^{\tau} \geqslant[x+y]_{\gamma^{\prime}}^{\tau}$ is true. Let $x \neq 0$ and consider any $\varepsilon>0$. We find from the definition of [ $]^{\tau}$, that. there exist $(c, Q) \in \mathscr{X}_{\mathscr{Y}}(x),(d, R) \in \mathscr{K}_{\mathcal{V}}(y)$ satisfying

$$
\sum_{i=1}^{2 k} c_{i} \tau\left(Q_{i}\right) \leqslant[x]_{\mathscr{V}}^{\tau}+\frac{1}{2} \varepsilon, \quad \sum_{i=1}^{2 k} d_{i} \tau\left(R_{i}\right) \leqslant[y]_{\mathscr{Y}^{\prime}}^{\tau}+\frac{1}{2} \varepsilon .
$$

Since $[x]_{\mathscr{V}}^{\tau}>0$ the set $\mathscr{J}=\left\{i \in\{1, \ldots, 2 k\} \mid c_{i}+d_{i}>0\right\}$ is non-empty. We have

$$
\begin{aligned}
{[x]_{\mathscr{V}}^{\tau}+[y]_{\mathscr{V}}^{\tau} } & \geqslant \sum_{i=1}^{2 k} c_{i} \tau\left(Q_{i}\right)+\sum_{i=1}^{2 k} d_{i} \tau\left(R_{i}\right)-\varepsilon \\
& =\sum_{i \in \mathscr{I}}\left(c_{i} \tau\left(Q_{i}\right)+d_{i} \tau\left(R_{i}\right)\right)-\varepsilon \\
& =\sum_{i \in \mathscr{J}}\left(c_{i}+d_{i}\right)\left(\frac{c_{i}}{c_{i}+d_{i}} \tau\left(Q_{i}\right)+\frac{d_{i}}{c_{i}+d_{i}} \tau\left(R_{i}\right)\right)-\varepsilon \\
& \geqslant \sum_{i \in \mathscr{J}}\left(c_{i}+d_{i}\right) \tau\left(\frac{c_{i}}{c_{i}+d_{i}} Q_{i}+\frac{d_{i}}{c_{i}+d_{i}} R_{i}\right)-\varepsilon \\
& =\sum_{i=1}^{2 k} \epsilon_{i} \tau\left(S_{i}\right)-\varepsilon
\end{aligned}
$$

where

$$
e_{i}= \begin{cases}c_{i}+d_{i} & \text { if } i \in \mathscr{I}, \\ 0 & \text { if } i \in\{1, \ldots, 2 k\}-\mathscr{J},\end{cases}
$$

and

$$
S_{i}= \begin{cases}\frac{c_{i}}{c_{i}+d_{i}} Q_{i}+\frac{d_{i}}{c_{i}+d_{i}} R_{i} & \text { if } i \in \mathscr{I}, \\ Q_{1} & \text { if } i \in\{1, \ldots, 2 k\}-\mathscr{I} .\end{cases}
$$


The reader can find that $(e, S) \in \mathscr{X}_{\mathscr{V}}(x+y)$ so that $[x]_{\mathscr{V}}^{\tau}+[y]_{\mathscr{V}^{\prime}}^{\tau} \geqslant[x+y]_{\mathscr{V}}^{\tau}-\varepsilon$ for any $\varepsilon>0$. Thus, the triangular inequality is valid. We have proved that []$_{\mathscr{V}}^{\tau}$ is a norm on $\mathbf{V}$.

Let $A \in \mathscr{O}$ and consider any $x \in \mathbf{V}$. It is easy to see that if $(c, Q) \in \mathscr{K}_{Y^{\prime}}(x)$ then $(c, A Q) \in \mathscr{K}_{Y}(A x)$, where

$$
A Q=\left(A Q_{1}, \ldots, A Q_{2 k}\right)
$$

Hence

$$
[A x]_{\mathscr{Y}}^{\tau} \leqslant \inf \left\{\sum_{i=1}^{2 k} c_{i} \tau\left(A Q_{i}\right) \mid(c, Q) \in \mathscr{X}_{Y^{\prime}}(x)\right\} \leqslant \tau(A)[x]_{\mathscr{Y}^{\prime}}^{\tau}
$$

by $(\beta)$. This completes the proof.

Theorem 1.2. Let $\tau$ be a nonnegative function on $d \gamma$ satisfying the conditions ( $\alpha$ ) and $(\beta)$, let $\|\mid\|$ be a norm on $\mathrm{V}$. Then:

(i) If

$$
\|x\| \leqslant\left(\max _{v \in Y^{\prime}}\|v\|\right)[x]^{\top}
$$

for any $x \in \mathbf{V}$ and for any finite subset $\boldsymbol{Y}^{\prime} \subset \mathbf{V}$ satisfying $\operatorname{Lin}\left(\mathscr{G} \mathscr{Y}^{\prime}\right)=\mathbf{V}$, then

$$
\sup _{\substack{x \in \mathbb{v} \\ x \neq 0}} \frac{\|A x\|}{\|x\|} \leqslant \tau(A)
$$

for all $A \in .(y$.

(ii) If

$$
\sup _{\substack{x \in \mathbf{V} \\ x \neq 0}} \frac{\|A x\|}{\|x\|} \leqslant \tau(A)
$$

for all $A \in G$, then

$$
\begin{aligned}
& {[]_{\mathcal{Y}^{\prime}}^{\tau} \text { is a norm on } \mathrm{V},} \\
& \|x\| \leqslant\left(\max _{v \in \mathcal{Y}^{\prime}}\|v\|\right)[x]_{\mathcal{Y}^{\prime}}^{\tau}
\end{aligned}
$$

and

$$
\sup _{\substack{x \in \mathbb{V} \\ x \neq 0}} \frac{[A x]^{\tau}}{[x]_{\gamma^{\prime}}^{\tau}} \leqslant \tau(A)
$$

for any finite subset $\mathscr{Y} \subset \mathbf{V}$ satisfying $\operatorname{Lin}(\circlearrowleft \mathbf{V})=\mathbf{V}$ and for all $x \in \mathbf{V}, A \in \leftrightarrow$. 
Proof. (i) Let $A \in \mathscr{W}$ and let $x \in \mathbf{V}-\{0\}$. Consider any finite set $\mathscr{V}=$ $\left\{v^{(1)}, \ldots, v^{(k)}\right\} \subset \mathbf{V}$ satisfying $\operatorname{Lin}\left(\mathscr{V}^{\mathcal{Y}^{\prime}}\right)=\mathbf{V}$ and put

$$
\vartheta=\|x\|\left(\max _{1 \leqslant i \leqslant k}\left\|v^{(i)}\right\|\right)^{-1} .
$$

Since $\mathscr{V} \neq\{0\}$ (because $\operatorname{Lin}\left(. \sigma^{\mathcal{Y}}\right)=V$ and $\left.\operatorname{dim} V \geqslant 1\right)$ and $x \neq 0$ we have $\vartheta \in$ $(0, \infty)$. Put $\mathscr{H}^{\prime}=\left\{w^{(1)}, \ldots, w^{(k)}, w^{(k+1)}\right\}$, where $w^{(1)}=v_{v^{(1)}}, \ldots, w^{(k)}=\vartheta_{l^{(k)}}$. $w^{(k+1)}=x$. It is obvious that $\operatorname{Lin}\left(\mathscr{\mathscr { V }} \mathscr{W ^ { \prime }}\right)=\mathbf{V}$. Further, let $c_{1}=0, \ldots, c_{k}=0$, $c_{k+1}=1, c_{k+2}=0, \ldots, c_{2(k+1)}=0$ and let $Q_{1}=A, \ldots, Q_{2(k+1)}=A$. We have

$$
\sum_{i=1}^{k+1}\left(c_{i} Q_{i}-c_{k+1+i} Q_{k+1+i}\right) w^{(i)}=Q_{k+1} w^{(k+1)}=A x,
$$

so that $\left(\left(c_{1}, \ldots, c_{2(k+1)}\right),\left(Q_{1}, \ldots, Q_{2(k+1)}\right)\right) \in \mathscr{K}_{\mathscr{Y}^{\prime}}(.1 x)$. Thus

$$
[A x]_{\mathscr{H}^{\prime}}^{\tau} \leqslant \sum_{i=1}^{2(k+1)} c_{i} \tau\left(Q_{i}\right)=\tau\left(Q_{k+1}\right)=\tau(A) .
$$

We summarize

$$
\frac{\|A x\|}{\|x\|} \leqslant \frac{1}{\|x\|}\left(\max _{w \in \mathscr{W}}\|w\|\right)[A x]_{\mathscr{W}^{\prime}}^{\tau}=\frac{1}{\|x\|}\|x\|[A x]_{\mathscr{H}^{\prime}}^{\tau} \leqslant \tau(A)
$$

(the first inequality holds by (3)).

(ii) Let $\boldsymbol{\gamma}^{\prime}=\left\{v^{(1)}, \ldots, v^{(k)}\right\} \subset \mathbf{V}, \operatorname{Lin}\left(\boldsymbol{c}^{\boldsymbol{V}^{\prime}}\right)=\mathbf{V}$. It is obvious that the set $\mathscr{I}=\left\{i \in\{1, \ldots, k\} \mid v^{(i)} \neq 0\right\}$ is non-empty. For any $x \in \mathbf{V},(c, Q) \in \mathscr{X}_{Y}(x)$ we have

$$
\begin{aligned}
\|x\| & =\left\|\sum_{i=1}^{k}\left(c_{i} Q_{i}-c_{k+i} Q_{k+i}\right) v^{(i)}\right\| \\
& =\left\|\sum_{i \in \mathscr{J}}\left(c_{i} Q_{i}-c_{k+i} Q_{k+i}\right) v^{(i)}\right\| \\
& \leqslant\left(\max _{i \in \mathscr{J}}\left\|v^{(i)}\right\|\right)\left(\sum_{i \in \mathscr{J}} c_{i} \frac{\left\|Q_{i} v^{(i)}\right\|}{\left\|v^{(i)}\right\|}+\sum_{i \in \mathscr{J}} c_{k+i} \frac{\left\|Q_{k+i} v^{(i)}\right\|}{\left\|v^{(i)}\right\|}\right) \\
& \leqslant\left(\max _{i \in \mathscr{I}}\left\|v^{(i)}\right\|\right)\left(\sum_{i \in \mathscr{J}} c_{i} \sup _{\substack{z \in \mathbb{V} \\
z \neq 0}} \frac{\left\|Q_{i} z\right\|}{\|z\|}+\sum_{i \in \mathscr{J}} c_{k+i} \sup _{\substack{z \in \mathbb{V} \\
z \neq 0}} \frac{\left\|Q_{k+i}\right\|}{\|z\|}\right) \\
(\text { by }(4)) & \leqslant\left(\max _{i \in \mathscr{J}}\left\|v^{(i)}\right\|\right)\left(\sum_{i \in \mathscr{J}} c_{i} \tau\left(Q_{i}\right)+\sum_{i \in \mathscr{J}} c_{k+i} \tau\left(Q_{k+i}\right)\right) \\
& \leqslant\left(\max _{1 \leqslant i \leqslant k}\left\|v^{(i)}\right\|\right)\left(\sum_{i=1}^{2 k} c_{i} \tau\left(Q_{i}\right)\right) .
\end{aligned}
$$


Hence

$$
\|x\| \leqslant\left(\max _{v \in \mathscr{V}}\|v\|\right)[x]_{\mathscr{V}}^{\tau}
$$

for all $x \in \mathrm{V}$. This inequality implies $[x]_{\mathscr{V}}^{\tau}>0$ for all $x \in \mathrm{V}-\{\mathbf{0}\}$. To complete the proof of (ii), it is sufficient to apply Theorem 1.1 .

Rem a rk. The condition (IV) is not essential for the validity of Theorems 1.1 and 1.2 as can be observed from their proofs.

The following characterization of the inverse matrix can be set as an example of the use of Theorem 1.2 .

Corollary. Let \|\| be a norm on $\mathbb{R}^{n}$, let \|\|$_{1}$ be the $l_{1}$-norm on $\mathbb{R}^{n}$, let $U$ be a real regular $n \times n$ matrix and let $u^{(1)}, \ldots, u^{(n)}$ be columns of $U$. Then

$$
\|x\| \leqslant\left(\max _{1 \leqslant i \leqslant n}\left\|u^{(i)}\right\|\right)\left\|U^{-1} x\right\|_{1}
$$

for each $x \in \mathbb{R}^{n}$.

Proof. Put $\mathbf{V}=\mathbb{R}^{n}, \boldsymbol{Y}^{\prime}=\left\{u^{(1)}, \ldots, u^{(n)}\right\}, \mathcal{C l}=\left\{\alpha I_{n} ; \alpha \in \mathbb{R}\right\}$ and let $\tau\left(\alpha I_{n}\right)=$ $|\alpha|$ for each $\alpha \in \mathbb{R}$. It is easy to see that the conditions (I), .., (IV) as wcll as $(\alpha)$ and $(\beta)$ are valid. The set $\boldsymbol{Y}^{\prime}$ is a base of $\mathbb{R}^{n}$, because the matrix $U$ is rrgular. It follows that $\operatorname{Lin}(A \mathscr{Y})=\mathbb{R}^{n}=\mathrm{V}$.

Let $x \in \mathbb{R}^{n}$. Put $a=\left(a_{1}, \ldots, a_{n}\right)^{T}=U^{-1} x$ and denote $a_{i}^{+}=\frac{1}{2}\left(\left|a_{i}\right|+a_{i}\right)$ and $a_{i}^{-}=\frac{1}{2}\left(\left|a_{i}\right|-a_{i}\right)$. Since

$$
\begin{aligned}
{[x]^{\tau}=} & \inf \left\{\sum_{i=1}^{2 n} c_{i} \tau\left(Q_{i}\right) \mid(c, Q) \in \mathscr{K}_{\mathcal{V}}(x)\right\} \\
= & \inf \left\{\sum_{i=1}^{2 n} c_{i}\left|\alpha_{i}\right| \mid c \in[0, \infty)^{2 n}, \alpha \in \mathbb{R}^{2 n}\right. \text { and } \\
& \left.c_{i} \alpha_{i}-c_{n+i} \alpha_{n+i}=a_{i} \text { for all } i=1, \ldots, n\right\} \\
\geqslant & \inf \left\{\sum_{i=1}^{n}\left|c_{i} \alpha_{i}-c_{n+i} \alpha_{n+i}\right| \mid c \in[0, \infty)^{2 n}, \alpha \in \mathbb{R}^{2 n}\right. \text { and } \\
& \left.c_{i} \alpha_{i}-c_{n+i} \alpha_{n+i}=a_{i} \text { for all } i=1, \ldots, n\right\} \\
= & \sum_{i=1}^{n}\left|a_{i}\right|=\left\|U^{-1} x\right\|_{1}=\sum_{i=1}^{n} a_{i}^{+}+a_{i}^{-}
\end{aligned}
$$

and

$$
\left(\left(a_{1}^{+}, \ldots, a_{n}^{+}, a_{1}^{-}, \ldots, a_{n}^{-}\right),\left(I_{n}, \ldots, I_{n}\right)\right) \in \mathscr{X}_{\mathscr{Y}}(x)
$$

we have $[x]_{\mathscr{Y}}^{\tau}=\left\|U^{-1} x\right\|_{1}$. Theorem 1.2(ii) completes the proof. 
Lemma 1.1. The set of is closed.

Proof. Consider any $A \in \mathscr{C}$. The condition (I) implies that $\{B-A ; B \in \mathscr{C}\}$ is a vector subspace of $\mathbf{M}_{n}$. Since $\mathbf{M}_{n}$ is a finite-dimensional vector space, the subspace $\{B-A ; B \in \mathscr{G}\}$ is closed. Thus $\mathscr{O}$ is a closed set as well.

Lemma 1.2. Let $\tau$ he a nonnegative function on of satisfying the conditions $(\delta)$ and $(\varepsilon)$. Let $\left\{a_{q}\right\}_{q=1}^{\infty}$ be a sequence of nonnegative real numbers and let $\left\{A_{q}\right\}_{q=1}^{\infty}$ be a convergent sequence of matrices of of such that

$$
\begin{aligned}
& \lim _{q \rightarrow \infty} a_{q} \tau\left(A_{q}\right)=0, \\
& \tau\left(\lim _{q \rightarrow \infty} A_{q}\right)=0, \\
& \tau\left(A_{q}\right)>0 \quad \text { for each } q \in \mathbb{N} .
\end{aligned}
$$

Then there exist a sequence $\left\{b_{r}\right\}_{r=1}^{\infty}$ of nonnegative real numbers, a convergent sequence $\left\{B_{r}\right\}_{r=1}^{\infty}$ of matrices of of and an increasing sequence $\left\{q_{r}\right\}_{r=1}^{\infty}$ of positive integers such that

$$
\begin{gathered}
\lim _{r \rightarrow \infty} b_{r} \tau\left(B_{r}\right)=0, \\
\tau\left(\lim _{r \rightarrow \infty} B_{r}\right)>0, \\
\tau\left(B_{r}\right)>0 \quad \text { for each } r \in \mathbb{N}, \\
b_{r} B_{r} x=a_{q_{r}} A_{q_{r}} x \quad \text { for each } r \in \mathbb{N}, x \in \mathbf{V} .
\end{gathered}
$$

Proof. Denote $A=\lim _{q \rightarrow \infty} A_{q}, \mathscr{Z}=\{Z \in \mathscr{S} \mid \tau(Z)=0\}$. By assumption, $A \in \mathscr{Z}$. First suppose that $\mathscr{Z}=\{A\}$. Put

$$
\alpha_{q}=\frac{1}{\left\langle\left\langle A-A_{q}\right\rangle\right\rangle}
$$

$\left(\tau\left(A_{q}\right)>0\right.$ and $\tau(A)=0$, thus $A_{q} \neq A$, hence $\left.\alpha_{q} \in(0, \infty)\right)$,

$$
\begin{aligned}
c_{q} & =\frac{a_{q}}{\alpha_{q}}, \\
C_{q} & =\alpha_{q} A_{q}+\left(1-\alpha_{q}\right) A
\end{aligned}
$$

for each $q \in \mathbb{N}$. We have $\left\langle\left\langle A-C_{q}\right\rangle\right\rangle=I$ for each $q \in \mathbb{N}$ and $C_{q} \in \mathscr{V}$ for each $q \in \mathbb{N}$ by condition (I). Hence, by Lemma 1.1, there exists a convergent subsequence $\left\{C_{q_{r}}\right\}_{r=1}^{\infty}$ such that $\lim _{r \rightarrow \infty} C_{q_{r}} \in G$. Denote $B=\lim _{r \rightarrow \infty} C_{q_{r}}$ and $b_{r}=c_{q_{r}}, B_{r}=C_{q_{r}}$ for each $r \in \mathbb{N}$. The sequence $\left\{B_{r}\right\}_{r=1}^{\infty}$ is convergent and $\left\{b_{r}\right\}_{r=1}^{\infty}$ is a sequence of nomnegative 
real numbers. Since $\left\langle\left\langle A-B_{r}\right\rangle\right\rangle=1$ for each $r \in \mathbf{N}$, we have $\langle\langle A-B\rangle\rangle=1$. Hence $B \notin\{A\}=\mathscr{Z}$, so that $\tau(B)>0$. The condition $(\varepsilon)$ implies $\tau\left(B_{r}\right)=\alpha_{q_{r}} \tau\left(A_{q_{r}}\right)$ and

$$
b_{r} \tau\left(B_{r}\right)=a_{q_{r}} \tau\left(A_{q_{r}}\right)
$$

for each $r \in \mathbf{N}$, hence $\tau\left(B_{r}\right)>0$ for each $r \in \mathbf{N}$ and $\lim _{r \rightarrow \infty} b_{r} \tau\left(B_{r}\right)=0$. The condition $(\delta)$ implies $b_{r} B_{r} x=a_{q_{r}} A_{q_{r}} x$ for each $r \in \mathbf{N}, x \in \mathbf{V}$. This completes the proof of the case $\mathscr{Z}=\{A\}$.

Now, let $\mathscr{Z} \neq\{A\}$. Put $\mathscr{Z}^{*}=\{Z-A ; Z \in \mathscr{Z}\}$. Then $\mathscr{Z}^{*}$ is a vector subspace of $\mathbf{M}_{n}$ by condition $(\varepsilon)$. Since $1 \leqslant \operatorname{dim} \mathscr{Z}^{*} \leqslant \operatorname{dim} \mathbf{M}_{n}=n^{2}$, there exists a finite orthonormal base $\left\{Y_{1}^{*}, \ldots, Y_{\nu}^{*}\right\}$ of $\mathscr{Z}^{*}$. Put

$$
\begin{aligned}
U_{q} & =A+\sum_{t=1}^{\nu}\left\langle A_{q}-A, Y_{t}^{*}\right\rangle Y_{t}^{*}, \\
\alpha_{q} & =\frac{1}{\left\langle\left\langle U_{q}-A_{q}\right\rangle\right\rangle}, \\
c_{q} & =\frac{a_{q}}{\alpha_{q}} \\
C_{q} & =\alpha_{q} A_{q}+\left(1-\alpha_{q}\right) U_{q}
\end{aligned}
$$

for each $q \in \mathbf{N}$. Since $\mathscr{Z}^{*}$ is a vector space, we have $U_{q}-A \in \mathscr{Z}^{*}$, i.e. $U_{q} \in \mathscr{Z}$ for each $q \in \mathbf{N}$. It implies $A_{q} \neq U_{q}$ for each $q \in \mathbf{N}$, because $\tau\left(A_{q}\right)>0$ for each $q \in \mathbb{N}$. Thus the numbers $\alpha_{q}$ are well-defined.

For each $q \in \mathbb{N}$, we have

$$
\begin{aligned}
\left\langle\left\langle A-C_{q}\right\rangle\right\rangle & =\left\langle\left\langle\alpha_{q} U_{q}-\alpha_{q} A_{q}+A-U_{q}\right\rangle\right\rangle \\
& \leqslant \alpha_{q}\left\langle\left\langle A_{q}-U_{q}\right\rangle\right\rangle+\left\langle\left\langle A-U_{q}\right\rangle\right\rangle \\
& =1+\left\langle\left\langle A-U_{q}\right\rangle\right\rangle
\end{aligned}
$$

and $\lim _{q \rightarrow \infty}\left\langle\left\langle A-U_{q}\right\rangle\right\rangle=0$ by the definition of the matrices $U_{q}$ and $A$. Thus, $\left\{C_{q}\right\}_{q=1}^{\infty}$ is a bounded sequence. It follows that there exists a convergent subsequence $\left\{C_{q_{r}}^{\prime}\right\}_{r=1}^{\infty}$. The condition (I) implies that $C_{q} \in \mathscr{Q}$ for each $q \in \mathbf{N}$. Thus, $\lim _{r \rightarrow \infty} C_{q_{r}} \in \mathscr{N}$ by Lemma 1.1. Put $B=\lim _{r \rightarrow \infty} C_{q_{r}}$ and $b_{r}=c_{q_{r}}, B_{r}=C_{q_{r}}$ for each $r \in \vec{N}^{\infty}$. The sequence $\left\{B_{r}\right\}_{r=1}^{\infty}$ is convergent and $\left\{b_{r}\right\}_{r=1}^{\infty}$ is a sequence of nonnegative real numbers. Since. 
for each $r \in \mathbb{N}$

$$
\begin{aligned}
U_{q_{r}}-A & =\sum_{t=1}^{\nu}\left\langle A_{q_{r}}-A, Y_{t}^{*}\right\rangle Y_{t}^{* *} \\
& =\alpha_{q_{r}} \sum_{t=1}^{\nu}\left\langle A_{q_{r}}-A, Y_{t}^{*}\right\rangle Y_{t}^{*}+\left(1-\alpha_{q_{r}}\right) \sum_{t=1}^{\nu}\left\langle A_{q_{r}}-A, Y_{t}^{*}\right\rangle Y_{t}^{* *} \\
& =\alpha_{q_{r}} \sum_{t=1}^{\nu}\left\langle A_{q_{r}}-A, Y_{t}^{*}\right\rangle Y_{t}^{*}+\left(1-\alpha_{q_{r}}\right) \sum_{t=1}^{\nu}\left\langle U_{q_{r}}-A, Y_{t}^{*}\right\rangle Y_{t}^{*} \\
& =\sum_{t=1}^{\nu}\left\langle B_{r}-A, Y_{t}^{*}\right\rangle Y_{t}^{*},
\end{aligned}
$$

$U_{q_{r}}-A$ is an orthogonal projection of $B_{r}-A$ to $\mathscr{Z}^{*}$ (see for example [5]). It follows that

$$
\begin{aligned}
\inf _{Z \in \mathscr{X}}\left\langle\left\langle B_{r}-Z\right\rangle\right\rangle & =\inf _{Z * \in \mathscr{X}_{*}^{*}}\left\langle\left\langle B_{r}-A-Z^{*}\right\rangle\right\rangle \\
& =\left\langle\left\langle B_{r}-A-\left(U_{q_{r}}-A\right)\right\rangle\right\rangle=1 .
\end{aligned}
$$

Hence,

$$
\inf _{Z \in \mathscr{X}}\langle\langle B-Z\rangle\rangle=1
$$

thus $B \notin \mathscr{Z}$, i.e. $\tau(B)>0$. The condition $(\varepsilon)$ implies $\tau\left(B_{r}\right)=\alpha_{q_{r}} \tau\left(A_{q_{r}}\right)$ and

$$
b_{r} \tau\left(B_{r}\right)=a_{q_{r}} \tau\left(A_{q_{r}}\right)
$$

for each $r \in \mathbb{N}$, hence $\tau\left(B_{r}\right)>0$ for each $r \in \mathbb{N}$ and $\lim _{r \rightarrow \infty} b_{r} \tau\left(B_{r}\right)=0$. The condition $(\delta)$ implies $b_{r} B_{r} x=a_{q_{r}} A_{q_{r}} x$ for each $r \in \mathbf{N}, x \in \mathbf{V}$. This completes the proof of the case $\mathscr{Z} \neq\{A\}$.

Now, we are able to formulate

Theorem 1.3. Let $\tau$ be a nonnegative function on of satisfying the conditions $(\alpha), \ldots,(\varepsilon)$ and let $\boldsymbol{\gamma}^{\prime}$ be a finite subset of $\mathrm{V}$ such that $\operatorname{Lin}\left(\mathscr{\alpha} \mathcal{Y}^{\prime}\right)=\mathrm{V}$. Then []$_{\mathcal{Y}}^{\tau}$ is a norm on $\mathbf{V}$ and

$$
\sup _{\substack{x \in \mathbf{V} \\ x \neq 0}} \frac{[A x]_{\mathcal{Y}}^{\tau}}{[x]_{\mathcal{V}}^{\tau}} \leqslant \tau(A)
$$

for all $A \in \mathscr{Q}$.

Proof. By Theorem 1.1, it is sufficient to show that $[x]^{\tau}>0$ for each $x \in$ $\mathbf{V}-\{\mathbf{0}\}$. Let us suppose that there exists $x \in \mathbf{V}-\{0\}$ such that $[x]^{\tau}=0$. Hence, 
there exists a sequence $\left(c^{(q)}, Q^{(q)}\right) \in \mathscr{X}_{\mathscr{Y}}(x), q \in \mathbb{N}$, such that

$$
\lim _{q \rightarrow \infty} \sum_{i=1}^{2 k} c_{i}^{(q)} \tau\left(Q_{i}^{(q)}\right)=0
$$

(onsider a matrix $Z \in \mathscr{W}$ such that $\tau(Z)=0$ (such a matrix does exist by condition $(\gamma))$. Put

$$
\begin{aligned}
\alpha_{i}^{(q)} & = \begin{cases}\frac{q^{-1}}{\left\langle\left\langle Q_{i}^{(q)}-Z\right\rangle\right\rangle} & \text { if } Q_{i}^{(q)} \neq Z, \\
1 & \text { if } Q_{i}^{(q)}=Z,\end{cases} \\
d_{i}^{(q, 0)} & =\frac{c_{i}^{(q)}}{\alpha_{i}^{(q)}}, \\
R_{i}^{(q, 0)} & =\alpha_{i}^{(q)} Q_{i}^{(q)}+\left(1-\alpha_{i}^{(q)}\right) Z
\end{aligned}
$$

for each $q \in \mathbb{N}, i \in\{1, \ldots, 2 k\}$. We have

$$
\left(d^{(q, 0)}, R^{(q, 0)}\right) \in \mathscr{K} \mathscr{w}(x) \text { for each } q \in \mathbb{N}
$$

hy condition $(\delta)$. Further, we have

$$
\sum_{i=1}^{2 k} d_{i}^{(q, 0)} \tau\left(R_{i}^{(q, 0)}\right)=\sum_{i=1}^{2 k} c_{i}^{(q)} \tau\left(Q_{i}^{(q)}\right) \text { for each } q \in \mathbb{N}
$$

by condition $(\varepsilon)$, hence

$$
\lim _{q \rightarrow \infty} \sum_{i=1}^{2 k} d_{i}^{(q, 0)} \tau\left(R_{i}^{(q, 0)}\right)=0
$$

Since $\lim _{q \rightarrow \infty}\left\langle\left\langle R_{i}^{(q, 0)}-Z\right\rangle\right\rangle=0$, the equality

$$
\lim _{q \rightarrow \infty} R_{i}^{(q, 0)}=Z
$$

is valid for each $i \in\{1, \ldots, 2 k\}$.

Let $\left\{\left(d^{(q, 1)}, R^{(q, 1)}\right)\right\}_{q=1}^{\infty}$ be an arbitrary subsequence of $\left\{\left(d^{(q, 0)}, R^{(q, 0)}\right)\right\}_{q=1}^{\infty}$ such that either

$$
\tau\left(R_{1}^{(q, 1)}\right)=0 \text { for all } q \in \mathbb{N}
$$

or

$$
\tau\left(R_{1}^{(q, 1)}\right)>0 \text { for all } q \in \mathbb{N}
$$


(recall that $\left.R^{(q, 0)}=\left(R_{1}^{(q, 0)}, \ldots, R_{2 k}^{(q, 0)}\right) \in \mathscr{c}^{2 k}, q \in \mathbf{N}\right)$. Now, construct step by set sequences $\left\{\left(d^{(q, 2)}, R^{(q, 2)}\right)\right\}_{q=1}^{\infty}, \ldots,\left\{\left(d^{(q, 2 k)}, R^{(q, 2 k)}\right)\right\}_{q=1}^{\infty}$ in the following way: if sequences $\left\{\left(d^{(q, 1)}, R^{(q, 1)}\right)\right\}_{q=1}^{\infty}, \ldots,\left\{\left(d^{(q, i)}, R^{(q, i)}\right)\right\}_{q=1}^{\infty}$ have already been constructed $(1 \leqslant i \leqslant 2 k-1)$, then let $\left\{\left(d^{(q, i+1)}, k^{(q, i+1)}\right)\right\}_{q=1}^{\infty}$ be an arbitrary subsequence of $\left\{\left(d^{(q, i)}, R^{(q, i)}\right)\right\}_{q=1}^{\infty}$ such that either

$$
\tau\left(R_{i+1}^{(q, i+1)}\right)=0 \quad \text { for all } q \in \mathbb{N}
$$

or

$$
\tau\left(R_{i+1}^{(q, i+1)}\right)>0 \text { for all } q \in \mathbb{N} .
$$

Thus, the sequence $\left\{\left(d^{(q, 2 k)}, R^{(q, 2 k)}\right)\right\}_{q=1}^{\infty}$ is a subsequence of $\left\{\left(d^{(q, 0)}, R^{(q, 0)}\right)\right\}_{q=1}^{\infty}$ and, for all $1 \leqslant i \leqslant 2 k$, we have either

$$
\tau\left(R_{i}^{(q, 2 k)}\right)=0 \text { for all } q \in \mathbb{N}
$$

or

$$
\tau\left(R_{i}^{(q, 2 k)}\right)>0 \text { for all } q \in \mathbb{N}
$$

Put

$$
\mathscr{I}=\left\{i \in\{1, \ldots, 2 k\} \mid \tau\left(R_{i}^{(q, 2 k)}\right)>0 \text { for all } q \in \mathbb{N}\right\}
$$

Suppose that $\mathscr{I}=\emptyset$. 'The condition $(\delta)$ implies that

$$
\sum_{i=1}^{2 k}\left(d_{i}^{(q, 2 k)} R_{i}^{(4,2 k)}-d_{k+i}^{(q, 2 k)} R_{k+i}^{(q, 2 k)}\right) v^{(i)}=0 \neq x
$$

for all $q \in \mathbf{N}$. This contradicts the relation $(6)$, hecause $\left\{\left(d^{(q, 2 k)}, R^{(q, 2 k)}\right)\right\}_{q=1}^{\sim}$ is a subsequence of $\left\{\left(d^{(q, 0)}, R^{(q, 0)}\right)\right\}_{q=1}^{\infty}$. Thus, $\mathscr{I} \neq \emptyset$.

Put

$$
\left\{\left(e^{(q, 0)}, S^{(q, 0)}\right)\right\}_{q=1}^{\infty}=\left\{\left(d^{(q, 2 k)}, R^{(q, 2 k)}\right)\right\}_{q=1}^{\infty} .
$$

Let $\left\{\left(e^{(q, 1)}, S^{(q, 1)}\right)\right\}_{q=1}^{\infty}$ be a subsequence of the seqcuence $\left\{\left(e^{(q, 0)}, S^{(q, 0)}\right)\right\}_{q=1}^{\infty}$ defined in the following way:

Put

$$
\left\{\left(e^{(q, 1)}, S^{(q, 1)}\right)\right\}_{q=1}^{\infty}=\left\{\left(e^{(q, 0)}, S^{(q, 0)}\right)\right\}_{q=1}^{\infty}
$$

if $1 \notin \mathscr{J}$. On the other hand, if $1 \in \mathscr{I}$. Lemma 1.2 applied for $a_{q}=c_{1}^{(y,())}$ and $A_{q}=S_{1}^{(q, 0)}$ generates the sequences $\left\{b_{r}\right\}_{r=1}^{\infty},\left\{B_{r}\right\}_{r=1}^{\infty}$ and $\left\{q_{r}\right\}_{r=1}^{\infty}$ (the assimuptions 
of Lemma 1.2 are valid by $(7),(8)$ and $(9))$; put

$$
\begin{aligned}
& e_{j}^{(r, 1)}= \begin{cases}e_{j}^{\left(q_{r}, 0\right)} & \text { if } j \neq 1, \\
b_{r} & \text { if } j=1,\end{cases} \\
& S_{j}^{(r, 1)}= \begin{cases}S_{j}^{\left(q_{r}, 0\right)} & \text { if } j \neq 1, \\
B_{r} & \text { if } j=1,\end{cases}
\end{aligned}
$$

for all $j=1, \ldots, 2 k$ and all $r \in \mathbf{N}$. Thus, if $\tau\left(S_{1}^{(q, 0)}\right)=0$ for all $q \in \mathbf{N}$, then

$$
\left\{\left(e^{(q, 1)}, S^{(q, 1)}\right)\right\}_{q=1}^{\infty}=\left\{\left(e^{(q, 0)}, S^{(q, 0)}\right)\right\}_{q=1}^{\infty}
$$

if $\tau\left(S_{1}^{(q, 0)}\right)>0$ for all $q \in \mathbb{N}$, then the entries $e_{1}^{\left(q_{r}, 0\right)}$ and $S_{1}^{\left(q_{r}, 0\right)}$ are redefined by

$$
e_{1}^{\left(q_{r}, 0\right)}=b_{r}, \quad S_{1}^{\left(q_{r}, 0\right)}=B_{r}
$$

and $\left\{\left(e^{(q, 1)}, S^{(q, 1)}\right)\right\}_{q=1}^{\infty}$ is the subsequence of the just modified $\left\{\left(e^{(q, 0)}, S^{(q, 0)}\right)\right\}_{q=1}^{\infty}$ determined by the sequence of indices $\left\{q_{r}\right\}_{r=1}^{\infty}$. Now construct step by step sequences $\left\{\left(e^{(q, 2)}, S^{(q, 2)}\right)\right\}_{q=1}^{\infty}, \ldots,\left\{\left(e^{(q, 2 k)}, S^{(q, 2 k)}\right)\right\}_{q=1}^{\infty}$ in the analogous way:

Suppose that $\left\{\left(e^{(q, 1)}, S^{(q, 1)}\right)\right\}_{q=1}^{\infty}, \ldots,\left\{\left(e^{(q, i)}, S^{(q, i)}\right)\right\}_{q=1}^{\infty}$ have already been constructed $(1 \leqslant i \leqslant 2 k-1)$. If $i+1 \notin \mathscr{J}$ then put

$$
\left\{\left(e^{(q, i+1)}, S^{(q, i+1)}\right)\right\}_{q=1}^{\infty}=\left\{\left(e^{(q, i)}, S^{(q, i)}\right)\right\}_{q=1}^{\infty} .
$$

On the other hand, if $i+1 \in \mathcal{I}$, Lemma 1.2 applied for $a_{q}=e_{i+1}^{(q, i)}$ and $A_{q}=S_{i+1}^{(q, i)}$ generates the sequences $\left\{b_{r}\right\}_{r=1}^{\infty},\left\{B_{r}\right\}_{r=1}^{\infty}$ and $\left\{q_{r}\right\}_{r=1}^{\infty}$ (the assumptions of Lemma 1.2 are valid by $(7),(8)$ and $(9))$; put

$$
\begin{aligned}
e_{j}^{(r, i+1)} & = \begin{cases}e_{j}^{\left(q_{r}, i\right)} & \text { if } j \neq i+1, \\
b_{r} & \text { if } j=i+1,\end{cases} \\
S_{j}^{(r, i+1)} & = \begin{cases}S_{j}^{\left(q_{r}, i\right)} & \text { if } j \neq i+1, \\
B_{r} & \text { if } j=i+1,\end{cases}
\end{aligned}
$$

for all $j=1, \ldots, 2 k$ and all $r \in \mathbf{N}$.

Let $\left\{\left(e^{(q)}, S^{(q)}\right)\right\}_{q=1}^{\infty}=\left\{\left(e^{(q, 2 k)}, S^{(q, 2 k)}\right)\right\}_{q=1}^{\infty}$. By the construction of the sequence $\left\{\left(e^{(q)}, S^{(q)}\right)\right\}_{q=1}^{\infty}$ (see Lemma 1.2) we have

$$
\begin{gathered}
\lim _{q \rightarrow \infty} e_{i}^{(q)} \tau\left(S_{i}^{(q)}\right)=0 \quad \text { for all } i \in \mathscr{J}, \\
\tau\left(\lim _{q \rightarrow \infty} S_{i}^{(q)}\right)>0 \quad \text { for all } i \in \mathscr{J}, \\
\tau\left(S_{i}^{(q)}\right)=0 \text { for all } q \in \mathbb{N}, \quad i \in\{1, \ldots, 2 k\}-\mathscr{J}, \\
\left(e^{(q)}, S^{(q)}\right) \in \mathscr{X}_{\mathscr{Y}}(x) \text { for each } q \in \mathbb{N} .
\end{gathered}
$$


Put $S_{i}=\lim _{q \rightarrow \infty} S_{i}^{(q)}$ for each $i \in \mathscr{J}$. Let $\gamma=\frac{1}{2} \min _{i \in \mathscr{I}} \tau\left(S_{i}\right)$. It is clear from (11) that $\gamma>0$. The conditions (I) and $(\alpha)$ imply that $\tau$ is a contimuons function on $s \gamma$. The continuity of $\tau$ and (11) imply the existence of a $q_{0} \in \mathbf{N}$ such that

$$
\tau\left(S_{i}^{(q)}\right)>\gamma>0 \text { for all } q \geqslant q_{0}, i \in \mathscr{I} .
$$

The relations (10) and (14) imply

$$
\lim _{q \rightarrow \infty} e_{i}^{(q)}=0 \text { for all } i \in \mathscr{J} .
$$

The condition $(\delta)$ and the relation (12) imply

$$
\sum_{\substack{i=1 \\ i \notin \mathscr{J}}}^{k} e_{i}^{(q)} S_{i}^{(q)} v^{(i)}-\sum_{\substack{i=k+1 \\ i \notin \mathscr{J}}}^{2 k} c_{i}^{(q)} S_{i}^{(q)} v^{(i-k)}=0
$$

for all $q \in \mathbf{N}$. Finally, (15) and (16) imply

$$
\begin{aligned}
\lim _{q \rightarrow \infty} & \sum_{i=1}^{2 k}\left(e_{i}^{(q)} S_{i}^{(q)}-e_{k+i}^{(q)} S_{k+i}^{(q)}\right) v^{(i)}= \\
& =\lim _{q \rightarrow \infty}\left(\sum_{\substack{i=1 \\
i \in \mathcal{J}}}^{2 k} e_{i}^{(q)} S_{i}^{(q)} v^{(i)}-\sum_{\substack{i=k+1 \\
i \in \mathcal{J}}}^{2 k} e_{i}^{(q)} S_{i}^{(q)} v^{(i-k)}\right) \\
& =\sum_{\substack{i=1 \\
i \in \mathscr{J}}}^{k} 0 \cdot S_{i} v^{(i)}-\sum_{\substack{i=k+1 \\
i \in \mathscr{J}}}^{2 k} 0 \cdot S_{i} v^{(i-k)}=0 \neq x,
\end{aligned}
$$

which contradicts (13). Thus, $[x]_{\mathscr{Y}}^{\top}>0$.

Theorem 1.4. Let $\|$ || he a norm on $\mathbf{V}$, let $\tau_{\|} \|$he a function on of definct as

$$
\tau_{\|\|}(A)=\sup _{\substack{x \in \mathfrak{x} \\ x \neq 0}} \frac{\|A x\|}{\|x\|}, \quad A \in \mathscr{V}
$$

and let $\mathscr{Y}$ be a finite sulset of $\mathrm{V}$ such that $\operatorname{Lin}\left(\mathcal{V}^{\prime} \mathcal{Y}^{\prime}\right)=\mathrm{V}$. Then []$_{Y^{\prime \prime}}^{\top} \|$ is a norm on $\mathrm{V}$,

$$
\|x\| \leqslant\left(\max _{v \in \mathscr{Y}^{\prime}}\|v\|\right)[x]_{\mathscr{Y}^{T}}^{T_{\|} \|}
$$


for all $x \in \mathbf{V}$ and

$$
\sup _{\substack{x \in \mathbb{v} \\ x \neq 0}} \frac{[A x]_{\mathscr{V}}^{\tau_{\|} \|}}{[x]_{\mathscr{V}}^{\tau_{\|}} \|} \leqslant \tau_{\|\|}(A)
$$

for all $A \in .{ }^{\prime}$.

Proof. Since the conditions $(\alpha), \ldots,(\varepsilon)$ hold for the function $\eta_{\|\|}((\gamma)$ holds according to (IV)), it is suflicient to apply Theorem 1.2 and Theorem 1.3.

\section{NONNEGATIVE FUNCTIONS ON STOCHASTIC MATRICES}

Troughout this section we assume $n \geqslant 2$. Define the vector space $\mathbf{V}$ by $\mathbf{V}=\{x \in$ $\left.\mathbf{R}^{n} \mid \mathbf{1}^{T} x=0\right\}$ and put $. \mathcal{Y}=\Sigma_{n}$. We observe that the condtions (I), .., (IV) are satisfied: the validity of (I) and (II) is obvious; (III) is true since $I_{n} \in \Sigma_{n}$; (IV) holds because $R \mathbf{V}=\{0\}$ for each $R \in \Sigma_{n}$ such that all columns of $R$ are identical.

Lemma 2.1. Let $\tau$ be a nonnegative function on $\Sigma_{n}$ satisfying the conditions $(\alpha), \ldots,(\varepsilon)$ and let $S \in \Sigma_{n}$. Then $\tau(S)=0$ if and only if all columns of $S$ are identical.

Proof. Let $\mathscr{R}_{n}$ be the set of all matrices of $\Sigma_{n}$ which have identical columms (i.e. $R \in \mathscr{P}_{n}$ iff $R \in \Sigma_{n}$ and there exists a vector $a \in \mathbf{R}^{n}$ such that $R=a 1^{T}$ ). It is easy to see that $R^{2}=R$ for each $R \in \mathscr{R}_{n}$. Hence

$$
\tau(R) \in\{0\} \cup[1, \infty) \text { for each } R \in \mathscr{R}_{n}
$$

by condition $(\beta)$.

Consider a matrix $Z \in \Sigma_{n}$ such that $\tau(Z)=0$ (the existence of such a matrix is guarenteed by condition $(\gamma))$. The form of the vector space $\mathbf{V}\left(\mathbf{V}=\left\{x \in \mathbb{R}^{n} \mid \mathbf{1}^{T} x=\right.\right.$ $0\}$ ) and condition $(\delta)$ imply that $Z \in \mathscr{R}_{n}$. Further, the function $\tau$ is continuous (by condition $(\alpha)$ ) and $\mathscr{R}_{n}$ is a convex set. We summarize that $\tau(R)=0$ for each $R \in \mathscr{R}_{n}$ by (17).

Finally, if $S \in \Sigma_{n}-\mathscr{R}_{n}$, then $\tau(S)>0$ by condition $(\delta)$. This completes the proof.

According to Lemma 2.1 we can replace the conditions $(\alpha), \ldots,(\varepsilon)$ by the following ones:

(S1) $\tau$ is a convex function on $\Sigma_{n}$;

(S2) $\tau\left(S_{1} S_{2}\right) \leqslant \tau\left(S_{1}\right) \tau\left(S_{2}\right)$ for all $S_{1}, S_{2} \in \Sigma_{n}$;

(S3) for each matrix $S \in \Sigma_{n}$ the equality $\tau(S)=0$ holds if and only if the matrix $S$ has identical colurnns; 
(S4) if $R, S \in \Sigma_{n}$ and if the matrix $R$ has identical columms then $\tau(\alpha S+(1-\alpha) R)=$ $\alpha \tau(S)$ holds for any $\alpha>0$.

Lemma 2.2. The equality $\Sigma_{n} \mathscr{Y}=\left\{x \in \mathbb{R}^{n} \mid \mathbf{1}^{T} x=0\right\}$ holds for each finite set $\mathscr{Y} \subset\left\{x \in \mathbf{R}^{n} \mid \mathbf{1}^{T} x=0, x \neq 0\right\}$.

Proof. It suffices to prove that

$$
\Sigma_{n}\{v\}=\left\{x \in \mathbf{R}^{n} \mid 1^{T} x=0\right\}
$$

for all $v \in\left\{x \in \mathbf{R}^{n} \mid \mathbf{1}^{T} x=0, x \neq \mathbf{0}\right\}$. Let

$$
v, x \in \mathbf{R}^{n}, \quad \mathbf{1}^{T} v=0, \quad \mathbf{1}^{T} x=0, \quad v \neq \mathbf{0} .
$$

Since $v \neq 0$, there exists an index $k$ such that $v_{k} \neq 0$. For all $i, j \in\{1, \ldots, n\}$ put

$$
s_{i j}= \begin{cases}\frac{1}{n} & \text { if } j \neq k, \\ \frac{1}{n}+\frac{x_{i}}{v_{k}} & \text { if } j=k .\end{cases}
$$

It is easy to see that $S=\left(s_{i j}\right)_{i, j=1}^{n} \in \Sigma_{n}$ and $S_{v}=x$.

Theorem 2.1. Let $\tau$ be a nonnegative function on $\Sigma_{n}$ satisfying the conditions (S1), .., (S4) and let $\boldsymbol{Y}^{\prime}$ be a finite subset of $\left\{x \in \mathbb{R}^{n} \mid \mathbf{1}^{T} x=0, x \neq 0\right\}$. Then [ ] ${ }^{\top}$ is a norm on the space $\left\{x \in \mathbb{R}^{n} \mid \mathbf{1}^{T} x=0\right\}$ and

$$
\sup _{\substack{x \in \mathbb{R}^{n}-\{0\} \\ \mathbf{1}^{T} x=0}} \frac{\left[P^{T} x\right]_{\mathscr{Y}}^{\tau}}{[x]^{\tau}} \leqslant \tau\left(P^{T}\right)
$$

for each $n \times n$ stochastic matrix $P$.

Pro of. If suffices to apply Theorem 1.3 and Lemmas 2.1 and 2.2 .

Theorem 2.2. Let $\tau$ be a nonnegative function on $\Sigma_{n}$ satisfying the conditions (S1), .., (S4) such that $\tau\left(P^{T}\right) \leqslant 1$ for each $n \times n$ stochastic matrix $P$. Then

$$
\tau\left(P^{T}\right) \geqslant \frac{1}{2} \max _{\substack{1 \leqslant i \leqslant n \\ 1 \leqslant j \leqslant n}} \sum_{k=1}^{n}\left|p_{i k}-p_{j k}\right|
$$

for each $n \times n$ stochastic matrix $P$. Moreover, for any finite set $\mathscr{Y} \subset\left\{x \in \mathbb{R}^{n} \mid\right.$ $\mathbf{1}^{T} x=0, x \neq 0$ \} there exists $a>0$ such that

$$
[x]_{Y^{r}}^{\tau}=\sum_{i=1}^{n} a\left|x_{i}\right|
$$


for each $x \in \mathbf{R}^{n}, \mathbf{1}^{T} x=0$.

Proof. This theorem follows from Theorem 2.1 and from [2, Theorem 3] (or from [3]).

Theorem 2.3. Let $\tau$ be a nonnegative function on $\Sigma_{n}$ satisfying the conditions (S1), .., (S4) and let $P$ be an $n \times n$ irreducible stochastic matrix. Then

$$
\max \{|\lambda| ; \lambda \in \sigma(P)-\{1\}\} \leqslant \tau\left(P^{T}\right) .
$$

Proof. This theorem follows from Theorem 2.1 and from [4, Theorem 3.1].

Lemma 2.3. Let $\tau_{1}, \tau_{2}$ he nonnegative functions on $\Sigma_{n}$ satisfying the conditions (S1), .., (S4). Then there exists $c>0$ such that $\tau_{1}\left(S^{\prime}\right) \leqslant c \tau_{2}\left(S^{\prime}\right)$ for all $S^{\prime} \in \Sigma_{n}$.

Proof. Let this propostition be not true, i.e., for each $q \in \mathbb{N}$ let there exist a matrix $S_{q} \in \Sigma_{n}$ such that

$$
\tau_{1}\left(S_{4}\right)>q \tau_{2}\left(S_{q}\right) .
$$

Lemma 2.1 implies that, for each $q \in \mathbb{N}, \tau_{1}\left(S_{q}\right)=0$ if and only if $\tau_{2}\left(S_{q}\right)=0$. Hence $\tau_{2}\left(S_{q}\right)>0$ for each $q \in \mathbf{N}$.

Consider any matrix $R \in \Sigma_{n}$ such that $R$ has identical columns. Let $\left\{\lambda_{q}\right\}_{q=1}^{\infty}$ be a sequence of positive real numbers such that

$$
\lim _{q \rightarrow \infty} \lambda_{q} S_{q}+\left(1-\lambda_{q}\right) R=R .
$$

For all $q \in \mathbf{N}$ put $a_{q}=1, A_{q}=\lambda_{q} S_{q}+\left(1-\lambda_{q}\right) R$. By Lemma 1.2 , there exist a sequence $\left\{b_{r}\right\}_{r=1}^{\infty}$ of positive real numbers (it is easy to see from the proof of Lemma 1.2 that if $a_{q}>0$ for all $q \in \mathbf{N}$ then $b_{r}>0$ for all $r \in \mathbf{N}$ ), a convergent sequence $\left\{B_{r}\right\}_{r=1}^{\infty}$ of matrices of $\Sigma_{n}$ and an increasing sequence $\left\{q_{r}\right\}_{r=1}^{\infty}$ of positive integers such that

$$
\begin{gathered}
\lim _{r \rightarrow \infty} b_{r} \tau_{i}\left(B_{r}\right)=0, \\
\tau_{i}\left(\lim _{r \rightarrow \infty} B_{r}\right)>0, \\
\tau_{i}\left(B_{r}\right)>0 \text { for each } r \in \mathbf{N}, \\
b_{r} B_{r} x=A_{y_{r}} x \text { for each } r \in \mathbf{N}, x \in \mathbf{V},
\end{gathered}
$$

where $i=1,2$ (it is easy to see from the proof of Lemma 1.2 that the sequences $\left\{b_{r}\right\}_{r=1}^{\infty},\left\{B_{r}\right\}_{r=1}^{\infty}$ and $\left\{q_{r}\right\}_{r=1}^{\infty}$ can coincide for both $\tau_{1}$ and $\tau_{2}$ ). Moreover, the equality (5) implies that

$$
b_{r} \tau_{i}\left(B_{r}\right)=a_{q_{r}} \tau_{i}\left(A_{q_{r}}\right) \quad \text { for all } r \in \mathbf{N}, i \in\{1,2\} .
$$


The condition (S4) implies

$$
\tau_{i}\left(A_{q_{r}}\right)=\lambda_{q_{r}} \tau_{i}\left(S_{q_{r}}^{\prime}\right) \text { for all } r \in \mathbf{N}, i \in\{1,2\} .
$$

We summarize that

$$
\begin{aligned}
\lim _{r \rightarrow \infty} \frac{\tau_{1}\left(B_{r}\right)}{\tau_{2}\left(B_{r}\right)} & =\lim _{r \rightarrow \infty} \frac{b_{r} \tau_{1}\left(B_{r}\right)}{b_{r} \tau_{2}\left(B_{r}\right)}=\lim _{r \rightarrow \infty} \frac{a_{q_{r}} \tau_{1}\left(A_{q_{r}}\right)}{a_{q_{r}} \tau_{2}\left(A_{q_{r}}\right)} \\
& =\lim _{r \rightarrow \infty} \frac{\tau_{1}\left(S_{q_{r}}^{\prime}\right)}{\tau_{2}\left(S_{q_{r}}\right)} \geqslant \lim _{r \rightarrow \infty} \frac{q_{r} \tau_{2}\left(S_{q_{r}}\right)}{\tau_{2}\left(S_{q_{r}}\right)}=\lim _{r \rightarrow \infty} q_{r}=\infty
\end{aligned}
$$

by (19) and (20). This contradicts (18), because the functions $\tau_{1}$ and $\tau_{2}$ are continuous by $(\mathrm{S} 1)$.

Theorem 2.4. Let $\tau$ be a nonnegative function on $\Sigma_{n}$ satisfying the conditions (S1), ..., (S4) and let $P$ be an $n \times n$ irreducible aperiodic stochastic matrix. P'ut $Q=P^{T}$. Then

$$
\begin{aligned}
\max \{|\lambda| ; \lambda \in \sigma(P)-\{1\}\} & =\lim _{k \rightarrow \infty}\left[\tau\left(Q^{k}\right)\right]^{1 / k} \\
& =\inf _{k \in \mathbb{N}}\left[\tau\left(Q^{k}\right)\right]^{1 / k} .
\end{aligned}
$$

Proof. Put $\xi(P)=\max \{|\lambda| ; \lambda \in \sigma(P)-\{1\}\}$. Since $P$ is an irreducible aperiodic stochastic matrix, we have

$$
\sigma\left(P^{k}\right)=\left\{\lambda^{k} ; \lambda \in \sigma(P)\right\},
$$

thus $\xi\left(P^{k}\right)=[\xi(P)]^{k}$. Hence $\xi(P) \leqslant\left[\tau\left(Q^{k}\right)\right]^{1 / k}$ for all $k \in \mathbf{N}$ by Theorem 2.3 .

Let \|\| be a norm on $\boldsymbol{R}^{n}$ and put

$$
\eta_{\|} \|\left(S^{\prime}\right)=\sup _{\substack{x \in \mathbf{y} \\ x \neq 0}} \frac{\|S x\|}{\|x\|}, \quad S \in \Sigma_{n},
$$

where $\mathbf{V}=\left\{x \in \mathbf{R}^{n} \mid \mathbf{1}^{T} x=0\right\}$. By Lemma 2.3 , there exists a number $c>0$ such that $\tau(S) \leqslant c \tau_{\|} \|(S)$ for all $S \in \Sigma_{n}$. It is proved in [4, Theorem 3.3] that

$$
\xi(P)=\lim _{k \rightarrow \infty}\left[\tau_{\|} \|\left(Q^{k}\right)\right]^{1 / k}=\inf _{k \in \mathbb{N}}\left[\tau_{\|\|}\left(Q^{k}\right)\right]^{1 / k} .
$$

Since $\lim _{k \rightarrow \infty} c^{1 / k}=1$, the proof is complete. 
Remark. Theorem 2.3 and Theorem 2.4 were proved in [4] for functions

$$
\tau_{\|\|}(P)=\sup _{\substack{x \in \mathbf{v} \\ x \neq \mathbf{0}}} \frac{\left\|x^{T} P\right\|}{\|x\|}, \quad P \in \mathscr{S}_{n},
$$

where \|\| is a norm on $\mathbf{R}^{n}$ and $\mathbf{V}=\left\{x \in \mathbf{R}^{n} \mid \mathbf{1}^{T} x=0\right\}$.

Examples. Put

$$
\begin{aligned}
\tau_{F}(S) & =\left(\sum_{i=1}^{n} \sum_{j=1}^{n}\left(s_{i j}-\frac{1}{n} \sum_{k=1}^{n} s_{i k}\right)^{2}\right)^{1 / 2}, \quad S \in \Sigma_{n}, \\
\tau_{m}(S) & =(n-1) \max _{\substack{1 \leqslant i \leqslant n-1 \\
1 \leqslant j \leqslant n-1}}\left|s_{i j}-s_{i n}\right|, \quad S \in \Sigma_{n}, \\
\tau_{s}(S) & =\sum_{i=1}^{n-1} \sum_{j=1}^{n-1}\left|s_{i j}-s_{i n}\right|, \quad S \in \Sigma_{n} .
\end{aligned}
$$

These function meet the demands of the conditions (S1), .., (S4). The function $\tau_{F}$ is called the Frobenius coefficient of ergodicity and is studied in [4] and [8].

The verification of (S1), (S3) and (S4) is easy. It is proved in [8] that (S2) holds true for $\tau_{F}$. We prove (S2) in the case of $\tau_{m}$ and $\tau_{s}$. Let

$$
S_{1}=\left(s_{i j}^{(1)}\right)_{i, j=1}^{n} \in \Sigma_{n}, \quad S_{2}=\left(s_{i j}^{(2)}\right)_{i, j=1}^{n} \in \Sigma_{n} .
$$

Since

$$
\begin{aligned}
\left|\sum_{k=1}^{n} s_{i k}^{(1)} s_{k j}^{(2)}-\sum_{k=1}^{n} s_{i k}^{(1)} s_{k n}^{(2)}\right| \\
\quad=\left|s_{i n}^{(1)}\left(s_{n j}^{(2)}-s_{n n}^{(2)}\right)+\sum_{k=1}^{n-1} s_{i k}^{(1)}\left(s_{k j}^{(2)}-s_{k n}^{(2)}\right)\right| \\
=\left|s_{i n}^{(1)} \sum_{k=1}^{n-1}\left(s_{k n}^{(2)}-s_{k j}^{(2)}\right)+\sum_{k=1}^{n-1} s_{i k}^{(1)}\left(s_{k j}^{(2)}-s_{k n}^{(2)}\right)\right| \\
=\left|\sum_{k=1}^{n-1}\left(s_{i k}^{(1)}-s_{i n}^{(1)}\right)\left(s_{k j}^{(2)}-s_{k n}^{(2)}\right)\right| \\
\leqslant \sum_{k=1}^{n-1}\left|s_{i k}^{(1)}-s_{i n}^{(1)}\right|\left|s_{k j}^{(2)}-s_{k n}^{(2)}\right|,
\end{aligned}
$$


we have

$$
\begin{aligned}
\tau_{m}\left(S_{1} S_{2}\right) & =(n-1) \max _{\substack{1 \leqslant i \leqslant n-1 \\
1 \leqslant j \leqslant n-1}}\left|\sum_{k=1}^{n} s_{i k}^{(1)} s_{k j}^{(2)}-\sum_{k=1}^{n} s_{i k}^{(1)} s_{k n}^{(2)}\right| \\
& \leqslant(n-1) \max _{\substack{1 \leqslant i \leqslant n-1 \\
1 \leqslant j \leqslant n-1}} \sum_{k=1}^{n-1}\left|s_{i k}^{(1)}-s_{i n}^{(1)}\right|\left|s_{k j}^{2}-s_{k n}^{(2)}\right| \\
& \leqslant(n-1)^{2} \max _{\substack{1 \leqslant i \leqslant n-1 \\
1 \leqslant j \leqslant n-1 \\
1 \leqslant k \leqslant n-1}}\left|s_{i k}^{(1)}-s_{i n}^{(1)}\right|\left|s_{k j}^{2}-s_{k n}^{(2)}\right| \\
& \leqslant(n-1)^{2} \max _{\substack{1 \leqslant i \leqslant n-1 \\
1 \leqslant k \leqslant n-1}}\left|s_{i k}^{(1)}-s_{i n}^{(1)}\right|_{\substack{1 \leqslant j \leqslant n-1 \\
1 \leqslant k \leqslant n-1}}\left|s_{k \cdot j}^{(2)}-s_{k n}^{(2)}\right| \\
& =\tau_{m}\left(S_{1}\right) \tau_{m}\left(S_{2}\right)
\end{aligned}
$$

and

$$
\begin{aligned}
\tau_{s}\left(S_{1} S_{2}\right) & =\sum_{i=1}^{n-1} \sum_{j=1}^{n-1}\left|\sum_{k=1}^{n} s_{i k}^{(1)} s_{k j}^{(2)}-\sum_{k=1}^{n} s_{i k}^{(1)} s_{k n}^{(2)}\right| \\
& \leqslant \sum_{i=1}^{n-1} \sum_{j=1}^{n-1} \sum_{k=1}^{n-1}\left|s_{i k}^{(1)}-s_{i n}^{(1)}\right|\left|s_{k j}^{(2)}-s_{k n}^{(2)}\right| \\
& =\sum_{j=1}^{n-1} \sum_{k=1}^{n-1}\left(\left|s_{k j}^{(2)}-s_{k n}^{(2)}\right| \sum_{i=1}^{n-1}\left|s_{i k}^{(1)}-s_{i n}^{(1)}\right|\right) \\
& \leqslant \sum_{j=1}^{n-1} \sum_{k=1}^{n}\left(\left|s_{k j}^{(2)}-s_{k n}^{(2)}\right| \sum_{g=1}^{n-1} \sum_{i=1}^{n-1}\left|s_{i j}^{(1)}-s_{i n}^{(1)}\right|\right) \\
& =\tau_{s}\left(S_{1}\right) \tau_{s}\left(S_{2}\right) .
\end{aligned}
$$

\section{References}

[1] R.A. Horn, Ch.R. Johnson: Matrix Analysis. Cambridge University Press, Cambridge, London, New York, New Rochelle, Melbourne and Sydney, 1986.

[2] A. Lesanovsky: Coefficients of ergodicity generated by non-symmetrical vector norms. Czechoslovak Math. J. 40 (115) (1990), 284-294.

[3] R. Kühne, A. Rhodius: $\Lambda$ characterization of Dobrushin's coeffiecient of ergodicity. Zeitschrift für Analysis und ihre Anwendungen 9 (2) (1990), 187-188.

[4] U.G. Rothblum, C.P. Tan: Upper bounds on the maximum modulus of subdominant eigenvalues of nonnegative inatrices. Linear Algebra Appl. 66 (1985), 45-86.

[5] W. Rudin: Real and Complex Analysis. McGraw-Hill. Inc. New York, 1974.

[6] E. Seneta: Coefficients of ergodicity: structure and applications. Adv. Appl. Prob. 11 (1979), 576-590. 
[7] E. Seneta: Spectrum localization by ergodicity coefficients for stochastic matrices. Linear and Multilinear Algebra 14 (1983), 343-347.

[8] E. Seneta, C.P. Tan: The Euclidean and Frobenius ergodicity coefficients and spectrum localization. Bull. Malaysia Math. Soc. (7) 1 (1984), 1-7.

[9] C.P. Tan: Coefficients of ergodicity with respect to vector norms. J. Appl. Prob. 20 (1983), 277-287.

[10] C.P. Tan: Spectrum localization of an ergodic stochastic matrix. Bull. Inst. Math. Acad. Sinica 12 (1984), 147-151.

[11] C.P. Tan: Spectrum localization using Hölder norms. Houston J. Math. 12 (1986), 441-449.

Author's address: Department of Probability and Statistics, Charles University, Sokolovská 83, 18600 Praha 8, Czech Republic. 